\title{
A Comparative Study of Myringoplasty Using Temporalis Fascia as a Graft Material by Underlay and Overlay Techniques
}

\author{
${ }^{1}$ Rahul T Thakur, ${ }^{2}$ SL Sonawale, ${ }^{3}$ Samir Joshi
}

\begin{abstract}
Introduction: Comparative study of myringoplasty (CSOM) with perforation is one of the common otological condition for which patients seek advice from otorhinolaryngologist.

Materials and methods: The study was carried out on patients diagnosed as CSOM in ENT department. Fifty patients were operated for myringoplasty with underlay (25) or overlay (25) technique with temporalis fascia as a graft material. The audiological evaluation was done after 3 months.
\end{abstract}

Results and observation: Total $82 \%$ cases showed improvement in hearing. The mean preoperative hearing loss was 35.90 ( $\pm 8.433 \mathrm{SD})$, and postoperative was 25.40 ( $\pm 5.962 S D)$ on paired t-test the differentiating factor was 8.832 and $p \leq 0.001$ which is statistically highly significant. Mean improvement in hearing using temporalis fascia and underlay technique was 9.8 $\mathrm{dB}( \pm 7.702 \mathrm{SD})$ and overlay technique was $11.2 \mathrm{db}( \pm 9.160 \mathrm{SD}$.

Conclusion: On applying paired t-test, the differentiating factor was 0.585 and $p=0.561$ which is statistically nonsignificant. Thus underlay and overlay technique of myringoplasty are equally effective.

Keywords: Myringoplasty, Overlay, Temporalis fascia, Underlay.

How to cite this article: Thakur RT, Sonawale SL, Joshi S. A Comparative Study of Myringoplasty Using Temporalis Fascia as a Graft Material by Underlay and Overlay Techniques. Int J Otorhinolaryngol Clin 2017;9(3):90-92.

Source of support: Nil

Conflict of interest: None

\section{INTRODUCTION}

Chronic suppurative otitis media is one of the common otological condition in India for which patients seek advice from an otorhinolaryngologist. Poverty, illiteracy, and overcrowding are all factors which play an important role in the causation of this disease. Various surgical modalities of treatment have been tried since ages, to eliminate the disease from middle ear cleft, with varying

${ }^{1}$ Assistant Professor, ${ }^{2}$ Associate Professor, ${ }^{3}$ Professor and Head

${ }^{1-3}$ Department of ENT, BJ Medical College, Pune, Maharashtra, India

Corresponding Author: Rahul T Thakur, Assistant Professor, Department of ENT, BJ Medical College, Pune, Maharashtra, India, e-mail: dr.rahul_thakur@yahoo.com

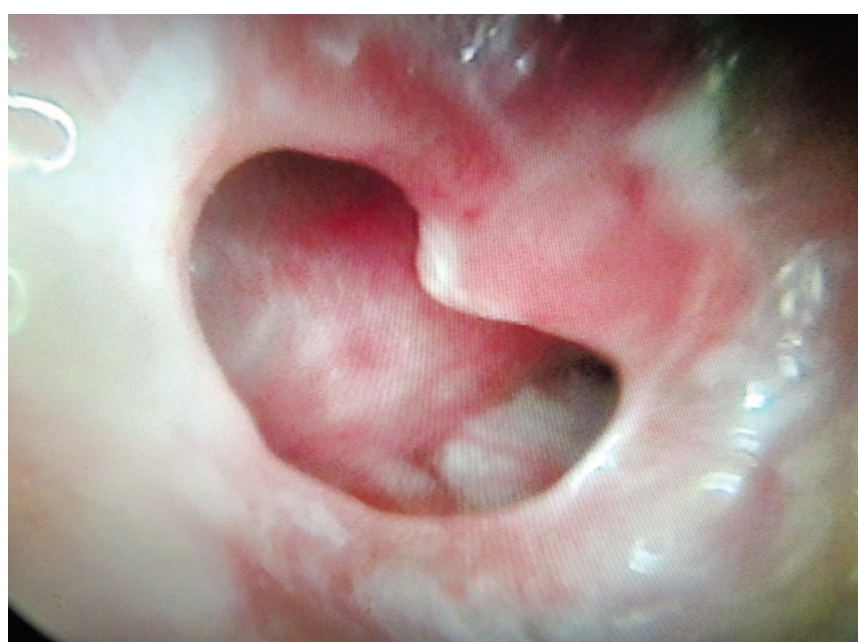

Fig. 1: Tympanic membrane perforation

degrees of success rate. One such modality of treatment is myringoplasty. The perforation (Fig. 1) seen in CSOM may be the only sequelae remaining when the pathological process in middle ear cleft has healed. ${ }^{1}$ It exposes the middle ear mucosa to an exogenous source of infection and also produces a conductive hearing loss, to address these issues the surgical technique of myringoplasty was developed, which consist of reconstruction of tympanic membrane perforation. Different materials have been used to reconstruct the tympanic membrane, most accepted of which is the temporalis fascia, because of its qualities of low metabolic rate, requiring lesser blood supply and is more resistant to infection. ${ }^{2}$ Myringoplasty is commonly performed by using underlay or overlay techniques. In the present study, an attempt is made to study the effectiveness of underlay and overlay technique with temporalis Fascia as graft material in and the improvement in hearing following its grafting.

\section{MATERIALS AND METHODS}

The present study was done in OPD of tertiary care hospital. Patients between $18-50$ years of age, dry ear ( 3 months) and safe type of CSOM were included in this study. Patients with ear discharge, unsafe CSOM, Immunocompromised status, patients with active TB were excluded from this study. All the patients underwent routine ENT evaluation in addition to which general medicine and anesthesia evaluation was carried out. Ear findings were noted with 


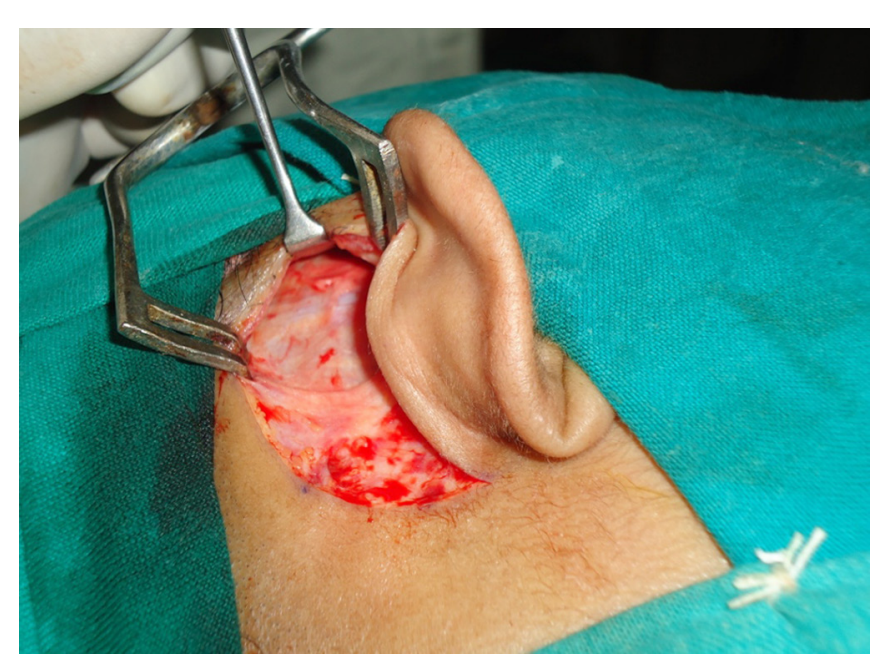

Fig. 2: Temporalis fascia graft harvesting

emphasis on size, site of perforation, margins of perforation, state of drum remnant, state of middle ear mucosa, presence or absence of discharge, tunning fork tests, pure tone audiometry. Preoperative assessment included ray mastoids, audiogram, and routine blood and urine investigations as per requirements for anesthesia. A majority of the patients underwent surgery under general anesthesia, while few were operated under local anesthesia ( $2 \%$ lignocaine +adrenaline) plus iv sedation. Autologous temporalis fascia graft was harvested (Fig. 2) through the postaural approach. The post canal wall based vascular tympanomeatal flap was raised, edges of perforation and the undersurface was freshened, the graft was secured either by underlay or overlay technique. The integrity of the ossicular chain was confirmed in all cases. Gel foam was used in the middle ear to support the graft kept in underlay fashion. The external auditory canal was packed with gel foam and ribbon gauze soaked with povidoneiodine solution. Post aural incision was sutured in layers, and the sterile mastoid dressing was given. Postoperative patients were given antibiotics, analgesics, antihistaminics sutures were removed on postoperative day 7 postoperative follow-up of patients were documented. The audiological evaluation was done postoperatively after 3 months. The outcome was taken as successful-if at the end of 3 months the graft was still in situ and acted as a scaffold for the epithelial healing and an effective hearing gain and failure-if there was graft rejection after 3 months or if there was no hearing gain.

\section{OBSERVATIONS AND RESULTS}

Total numbers of ears operated in this study were 50 . The age range was from 18 to 50 years. The mean age of the patients was 31 years. The male to female ratio was 29:21. There was no statistically significant sex or age predilection for the sample of 50 patients. Tympanoplasty was done with underlay 25 and overlay 25. Type of graft material used was temporalis fascia for all the cases. Postaural was the preferred route for all the cases. Forty-one cases were operated under general anesthesia, 41 and 9 cases in local anesthesia. In our study of 50 cases, 25 cases were operated with underlay technique out of which 21 cases had positive graft uptake while four had negative graft uptake. Twenty-five cases were operated with overlay technique out of which 20 cases had positive graft uptake while five had negative graft uptake. On applying Chi-square test the differentiating factor $=1$ and the $p$ value is 1.0 which is statistically nonsignificant. In our study 5 cases (10\%) had hearing loss of $20-25 \mathrm{db}, 17$ cases (34\%) had hearing loss of $26-30 \mathrm{db}, 13$ cases $(26 \%)$ had hearing loss of 31-35 db, 4 cases (08\%) had hearing loss of $36-40 \mathrm{db}$, 4 cases (8\%) had hearing loss of $41-45 \mathrm{db}, 4$ cases $(08 \%)$ had hearing loss of $46-50 \mathrm{db}$ and 3 cases $(6 \%)$ had hearing loss of 51-55db. In our study 6 cases that were operated with underlay technique and 4 cases that were operated with overlay technique were benefited with 1-5 $\mathrm{db}$ closure of air bone gap, 9 cases who were operated with underlay technique and 7 cases who were operated with overlay technique were benefited with 6-10 db closure of air bone gap. Two cases that were operated with underlay technique and 4 cases that were operated with overlay technique were benefited with $11-15 \mathrm{db}$ closure of air bone gap. Two cases that were operated with underlay technique and 1 case who were operated with overlay technique were benefited with 16-20 db closure of air bone gap. One case that was operated with underlay technique and 2 cases that were operated with overlay technique were benefited with $21-25 \mathrm{db}$ closure of air bone gap. One case that was operated with underlay technique and 2 cases that were operated with overlay technique were benefited with 26-30 $\mathrm{db}$ closure of air bone gap. Four cases that were operated with underlay technique and 5 cases that were operated with overlay technique showed no benefit with closure of air bone gap (Graph 1).

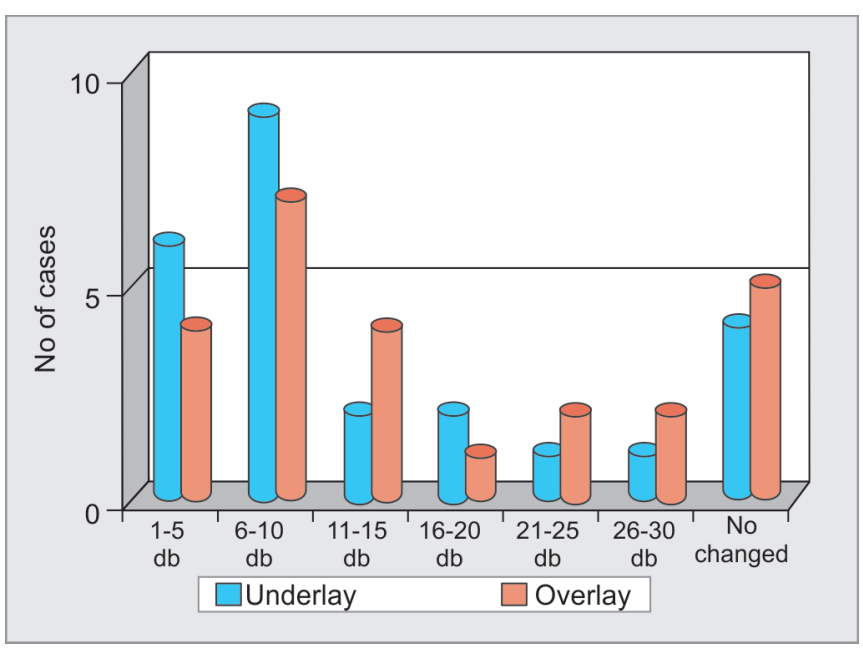

Graph 1: Technique-wise improvement in hearing 
Out of the 50 operated cases the mean preoperative air-bone gap was $35.90( \pm 8.433 \mathrm{SD})$ while the postoperative air bone gap closure was $25.40( \pm 5.962 \mathrm{SD})$. On applying paired $\mathrm{t}$ test the differentiating factor is 8.832 and $p \leq 0.001$ which is highly significant. Out of the 50 operated cases 25 cases were operated with underlay technique and 25 cases were operated with overlay technique the mean difference between preoperative air-bone gap and postoperative air-bone gap closure was 9.8 $( \pm 7.702 \mathrm{SD})$ with underlay technique and $11.2( \pm 9.160 \mathrm{SD})$ for overlay technique .on applying unpaired $t$ test the differentiating factor is 0.585 and $p=0.561$ which is statistically not significant.

\section{DISCUSSION}

This is a study of 50 myringoplasties on 50 patients between ages $15-50$ years. Conservative measures were first tried in all cases these include antibiotics, repeated aural toilets. The patient population attending our hospital was mostly from low socioeconomic status. Many had poor personal hygiene and poor nutritional status. These were probably some of the factors which contributed to the higher rate of graft rejection. The cases which developed complications were either treated with application of TCA if the residual perforation is small or were considered for second surgery. Michael and Glasscock et al. ${ }^{3}$ report in their study of 1556 tympanic membrane grafting that there was no difference in take rate of graft based upon the age of the patient. In our study, the age of patient varied between 18 years and 46 years; the take-up rate of graft for different age group was the same, which suggests that age did not make any difference intake rate. The mean age for underlay technique of myringoplasty was $34.24(S D \pm 9.435)$ and that for overlay was 31.84 (SD \pm 6.615$)$. With a $p$ value of 0.796 . Which is statistically nonsignificant. This is in keeping with observations of the existing literature. Browning ${ }^{4}$ states that there is a close relationship between chronic otitis medium and low socieconomic group. Higher incidence is because of poor general health, malnutrition and overcrowding. In the present study of 50 cases, 30 cases $(60 \%)$ belonged to low income group and 20 cases (40\%) belonged to medium income group. Low income group in our country is associated with ignorance and illiteracy, and the thus there is more prevalence of chronic otitis media in this group. Thomas et al. ${ }^{5}$ in their study of 26 cases, male to female ratio was 1.36. Mathai ${ }^{6}$ in his study of 200 cases, the male to female ratio was 1.85 . In our present study of 50 cases, we had 21 females and 29 males who underwent myringoplasty, with male to female ratio of 1:1.38, This in keeping with the observations of existing literature. Glasscock et al. ${ }^{7}$ in study of myringoplasty with temporalis fascia using underlay and overlay technique demonstrated that there is no significant difference between techniques. Singh et al. ${ }^{8}$ in comparative study of the underlay and overlay techniques of myringoplasty in large and subtotal perforations of the tympanic membrane demonstrated that there is no significant difference between the techniques.

This is in keeping with the observation of existing literature. We found that overall satisfactory hearing outcome can be achieved irrespective of the technique done for grafting although difference not being statistically significant.

\section{SUMMARY AND CONCLUSION}

Several factors govern the results of myringoplasty. Although in the modern era surgical success rate of myringoplasty is crossing the previously considered optimum of $90 \%$, it has never reached $100 \%$ even in very experienced hands. We found that the results of surgery in terms of graft uptake were numerically better in the group with underlay technique than overlay technique by $2 \%$. Though the difference is statistically not significant. Also, there is no statistically significant difference in hearing gain of both the techniques. Thus to conclude, both underlay and overlay techniques are excellent methods of myringoplasty. Graft uptake is suitable for both with slightly better take rate for underlay. Both techniques give good hearing improvement. Although 50 cases are by no means a small figure, we propose a larger multicentric study to throw more light on the topic.

\section{REFRENCES}

1. Palva T, Virtanen H. Pitfalls in myringoplasty. Acta Otolaryngology 1982;93:441-446.

2. Sheehy JL, Glasscock ME. Tympanic membrane grafting with temporalis fascia. Archives of Otolaryngology 1967;86:391-402.

3. Otorhinolaryngology Head and Neck Surgery By-John Jacob Ballenger, 15th Edition, pp. 1025.

4. Browning GG. Aetiopathology of inflammatory conditions of the external and middle ear. 6th ed. In: Scott Brown's Otolaryngology, Booth JB, ed. Oxford: Butter Heineworth Publication 1997;3:1-37.

5. Thomas CP, Nielsen RT, Tos M. Bilateral myringoplasty in chronic otitis media. Laryngoscope 2007;117:903-906.

6. Mathai J. Myringoplasty with temporalis fascia: Analysis of 200 cases. Indian Journal of Otology and Head and Neck Surgery 1999;51(2):9-13.

7. Glasscock ME III. Tympanic membrane grafting with fascia: overlay vs. undersurface technique. Laryngoscope 1973; 83:754-770.

8. Singh M, Rai A, Bandyopadhyay S, et al. Comparative study of the underlay and overlay techniques of myringoplasty in large and subtotal perforations of the tympanic membrane. J Laryngol Otol 2003;117:444-448. 